\title{
Endothelial Rbpj is essential for the education of tumour-associated macrophages
}

Ronja Mülfarth ${ }^{1,2^{\star}}$, Elisenda Alsina-Sanchis ${ }^{1^{\star}}$, Iris Moll ${ }^{1}$, Sarah Böhn ${ }^{1}$, Lena Wiedmann ${ }^{1,2}$ Lorea Jordana ${ }^{1}$, Tara Ziegelbauer ${ }^{1}$, Jacqueline Taylor ${ }^{1}$, Francesca De Angelis Rigotti ${ }^{1,3}$, Adrian Stögbauer ${ }^{1}$, Benedetto Daniele Giaimo ${ }^{4}$, Adelheid Cerwenka ${ }^{5,6}$, Tilman Borggrefe ${ }^{4}$, Andreas Fischer ${ }^{1,5,7, *}$, Juan Rodriguez-Vita ${ }^{1,3, *}$

${ }^{1}$ Division Vascular Signaling and Cancer, German Cancer Research Center (DKFZ), 69120 Heidelberg, Germany.

${ }^{2}$ Faculty of Biosciences, University of Heidelberg, 69120 Heidelberg, Germany.

${ }^{3}$ Tumour-Stroma Communication Laboratory, Centro de Investigación Príncipe Felipe, 46012 Valencia, Spain.

${ }^{4}$ Institute of Biochemistry, University of Giessen, 35392 Giessen, Germany.

${ }^{5}$ European Center for Angioscience (ECAS), Medical Faculty Mannheim, University of Heidelberg, 68167 Mannheim, Germany.

${ }^{6}$ Department of Immunobiochemistry, Mannheim Institute for Innate Immunoscience (MI3), Medical Faculty Mannheim, University of Heidelberg, 68167 Mannheim, Germany

${ }^{7}$ Institute for Clinical Chemistry, University Medical Center Göttingen, 37075 Göttingen, Germany.

* Equal contribution.

Correspondence to: a.fischer@dkfz.de, rodriguez@cipf.es 


\section{Abstract}

2 Epithelial ovarian cancer (EOC) is one of the most lethal gynaecological cancers worldwide.

3 EOC cells educate tumour-associated macrophages (TAMs) through CD44-mediated

4 cholesterol depletion to generate an immunosuppressive tumour microenvironment (TME).

5 In addition, tumour cells frequently activate Notch1 receptors on endothelial cells (ECs) to

6 facilitate metastasis. However, little is known whether the endothelium would also influence

7 the education of recruited monocytes. Here, we report that canonical Notch signalling

8 through RBPJ in ECs is an important player in the education of TAMs and EOC progression.

9 Deletion of $R b p j$ in the endothelium of adult mice reduced infiltration of monocyte-derived

10 macrophages into the TME of EOC and prevented the acquisition of a typical TAM gene

11 signature. This was associated with stronger cytotoxic activity of $\mathrm{T}$ cells and decreased

12 tumour burden. Mechanistically, we identified CXCL2 as a novel Notch/RBPJ target gene.

13 This angiocrine factor regulates the expression of CD44 on monocytes and subsequent

14 cholesterol depletion of TAMs. Bioinformatic analysis of ovarian cancer patient data showed

15 that increased CXCL2 expression is accompanied by higher expression of CD44 and TAM

16 education. As such, EOC cells employ the tumour endothelium to secrete CXCL2 in order to

17 facilitate an immunosuppressive microenvironment.

\section{Introduction}

High grade serous ovarian cancer is the deadliest type of all gynaecological

21 cancers $^{1}$. The high mortality rate is due to the fact that most women have already developed

22 peritoneal metastasis at the time of diagnosis. Epithelial ovarian cancer (EOC) cells can

23 directly infiltrate the peritoneal cavity to seed metastases, a process called transcoelomic

24 metastasis $^{2}$. Metastatic EOC cells initially reside in the omentum, where they undergo

25 certain adaptations allowing them to spread throughout the whole peritoneal cavity. 
1 Importantly, this peritoneal microenvironment is so immunosuppressive that even the

2 infiltration of effector $T$ cell does not per se correlate with better prognosis ${ }^{3}$.

Peritoneal spread of tumour cells is accompanied by monocyte-derived macrophage

4 (MN-derived macrophages) recruitment, which eventually become the most abundant

5 myeloid cell type ${ }^{4}$ and are a major contributor to the immunosuppressive TME in $\mathrm{EOC}^{3}$.

6 Upon recruitment from blood to the tumour, monocytes differentiate into macrophages which

7 are further educated by the TME. Eventually, tumour-associated macrophages (TAMs)

8 strongly promote the progression of metastatic ovarian cancer ${ }^{5}$. However, little is known

9 about the contribution of other stromal cells to the peritoneal spread of EOC cells and to 10 macrophage education.

Monocytes must cross the vascular endothelial barrier before infiltrating peritoneal organs or the peritoneal fluid. Endothelial cells (ECs) not only form tubes for the transport of

13 blood, but also produce soluble factors controlling the differentiation and function of adjacent cells. These angiocrine functions are highly context and organ specific ${ }^{6,7}$. In cancer, ECs provide angiocrine factors which influence tumour progression ${ }^{8}$. Therefore, we hypothesized that monocytes are influenced by ECs, for instance during transmigration, while infiltrating into peritoneal tissues.

Notably, tumours can alter the transcriptome of $\mathrm{ECs}^{9,10}$ and this may also influence transmigrating monocytes. For example, endothelial Notch signalling activity is frequently higher in tumours like EOC and in the metastatic niche compared to ECs from non-

21 tumourous tissue ${ }^{11}$. Notch signalling is a highly conserved cell-to-cell communication system. Ligand binding induces cleavage of the transmembrane Notch receptors releasing the intracellular domain (ICD) which enters the nucleus to alter gene transcription. This canonical signalling pathway relies on the DNA-binding protein RBPJ, which turns into a 25 transcriptional activator upon binding of a Notch receptor $I C D^{12}$. Sustained endothelial 26 Notch1 signalling is associated with increased myeloid cell infiltration and metastasis ${ }^{11}$. The 27 Notch pathway in ECs is a major regulator of angiogenesis, metabolism, angiocrine 
1 functions and tumour cell transmigration ${ }^{11,13-18}$. Although EOC cells do not necessarily have

2 to cross the endothelial barrier to spread throughout the peritoneum we hypothesized that

3 endothelial Notch signalling could still influence EOC progression via transmigrating myeloid

4 cells.

5 Here, we provide insights into the essential role of RBPJ in ECs for the recruitment of

6 monocytes to the microenvironment of metastatic EOC and their proper education into pro-

7 tumoural TAMs.

9 Results

10 Deletion of $R b p j$ in endothelial cells reduces EOC progression

11 Metastatic EOC cells seed initially in the omentum and later spread within the 12 peritoneum. During these steps, tumour cells undergo transcriptional changes that allow 13 them to further grow and colonise (Fig. 1a). The latter is strongly influenced by MN-derived 14 macrophages ${ }^{19}$. To determine the contribution of canonical Notch signalling in ECs to 15 myeloid cell infiltration and EOC progression, we used the tamoxifen-inducible VE-Cadherin 16 (Cdh5) Cre ${ }^{\mathrm{ERT} T 2}$ strain to delete Rbpj specifically in ECs of adult mice (Rbpj $\left.{ }^{\mathrm{i \Delta EC}}\right)$. This mouse 17 model is well established and leads to robust gene recombination in ECs of several $18 \operatorname{organs}^{14,15,17,18}$ (Fig. 1b). Under physiological conditions, there were no differences in blood vessel density in the larger omentum upon Rbpj deletion in ECs compared to controls (Suppl. Fig. 1a,b). However, upon intraperitoneal injection of ID8 cells mimicking metastatic

21 EOC, the omenta showed evidence of tumour nodule growth (Fig. 1c) and had significantly 22 higher vessel density in $R b p j^{i \Delta E C}$ mice compared to control animals (Fig. 1d). The density of 23 endothelial coverage with a-smooth muscle actin-positive mural cells was, however, 24 unchanged (Suppl. Fig. 1c). Despite increased vessel density, tumour burden in omenta of $25 R b p j^{i \Delta E C}$ mice was significantly lower than that in their littermate controls (Fig. 1e). Moreover, 
1 peritoneal spread of tumour cells was significantly reduced as well in $R b p j^{i \Delta E C}$ mice (Fig. 1f)

2 concluding that deletion of Rbpj in ECs reduces EOC progression and metastasis.

4 Deletion of endothelial $R b p j$ decreases monocyte-derived macrophage recruitment

5 Next, we analysed the immune cell compartment of our EOC model since it is a

6 major contributor to tumour progression. High Notch signalling activity in ECs induces

7 VCAM1 expression, which promotes leukocyte extravasation ${ }^{11,20}$. However, VCAM1

8 expression was unchanged between $R b p j^{\mathrm{i} \Delta \mathrm{EC}}$ and their controls littermates (Suppl. Fig. 2a).

9 Nevertheless, whole-mount staining of omenta revealed that there was a reduction in

10 immune cells inside tumour nodules of $R b p j^{i \Delta \mathrm{EC}}$ mice compared to controls (Fig. $2 \mathrm{a}$ and

11 Suppl. Fig. 2b). Interestingly, tumours in control mice contained more cells with a large

12 vacuolated cytoplasm, reminiscent of macrophages. Therefore, we analysed this cell

13 population in greater detail. Tumour-bearing omenta from $R b p j^{i \Delta \mathrm{EC}}$ mice contained less

$14 \mathrm{CD}_{11 \mathrm{~b}^{+}}$cells within tumour nodules, although this reduction was not statistically significant

15 (Fig. 2b). In order to better determine the macrophage subpopulations responsible for the

16 observed decrease in CD11 $\mathrm{b}^{+}$cells; we studied the myeloid and macrophage populations in

17 the peritoneal cavity to understand whether RBPJ in ECs could play a role in their

18 composition. After four weeks of tumour growth, there were no significant differences in the

19 total amount of myeloid cells or macrophages (Fig. 2c,d). However, we found decreased

20 numbers of small peritoneal macrophages (SPMs), which are MN-derived macrophages

21 characterized as MHC-II $/$ F4/80 low (Fig. 2e) and CCR2 ${ }^{+} / \mathrm{Tim}^{-}$(Fig. 2f). Notably, in naïve

22 (tumour-free) conditions, endothelial Rbpj deletion had no effect on peritoneal macrophage

23 populations (Suppl. Fig. 2c-f). These data suggest that the tumour-driven recruitment of

24 monocytes into the peritoneum is impaired upon endothelial deletion of $R b p j$.

To further dissect endothelial chemotaxis in a more simplified in vitro system, we

26 switched to the human system and measured transmigration of human CD14 ${ }^{+}$monocytes 
1 through a monolayer of human ECs in a transwell insert. Chemotaxis was stimulated by SK-

2 OV-3 human ovarian cancer cells below the insert (Fig. $\mathbf{2 g}$ ). RBPJ deletion in human

3 umbilical vein ECs (HUVECs) resulted in significantly decreased monocyte transmigration

4 rates compared to control HUVECs (Fig. 2h). This further suggests that canonical Notch

5 signalling in ECs is important to facilitate monocyte recruitment into the TME.

7 Canonical Notch signalling in ECs regulates monocyte recruitment through CXCL2

8 We have previously analysed the transcriptional induction of chemokines in HUVECs 9 upon Notch1 ICD (N1ICD) overexpression ${ }^{11}$. Based on this, we transduced RBPJ-deficient 10 HUVEC and control cells with N1ICD to determine which of those transcriptional changes 11 require RBPJ. The chemokines that were induced by N1ICD through RBPJ were CCL1, 12 CCL21, CXCL12, and CXCL2 (Fig. 3a) and we excluded those that were not induced 13 through RBPJ (Suppl. Fig. 3a-b). Next, we measured the mRNA expression levels of these 14 chemokines in peritoneal adipose tissue obtained from $R b p j^{i \Delta \mathrm{EC}}$ mice. This revealed that 15 there was lower $C x C / 2$ expression in peritoneal adipose tissue from $R b p j^{i \Delta E C}$ mice compared 16 to littermate controls, whereas the other chemokines analysed were not changed (Fig. $\mathbf{3 b}$ ). 17 Analysis of the same cytokines in endothelial-specific N1ICD mice ${ }^{11,15}$, exposed that higher endothelial Notch1 signalling activity led to higher $\mathrm{Cxcl} 2$ expression in peritoneal adipose tissue (Fig. 3c).

In silico analysis showed that the mouse and human Cxcl2 gene contains RBPJbinding sites in the promoter region (Fig. 3d). Consistently, cultured human ECs secreted higher CXCL2 protein levels after being transduced with N1ICD expressing adenovirus compared to GFP-transduced control cells (Fig. 3e).

These data revealed that canonical Notch signalling induces CXCL2 expression in

25 ECs. CXCL2 is known to attract granulocytes but also to a lesser extend monocytes ${ }^{21}$. As 26 such, it poses as an interesting endothelial Notch target, which could mediate the observed 
1 effects on monocyte recruitment into the TME. To evaluate whether CXCL2 was capable of

2 attracting monocytes, we performed transwell chemotaxis experiments and observed that

3 recombinant CXCL2 induced monocyte chemotaxis (Fig. 3f). Next, we silenced CXCL2

4 expression in ECs using shRNA, which led to an about $50 \%$ reduction of CXCL2 protein

5 expression (Fig. $\mathbf{3 g}$ ). Compared to non-silencing control, this reduction of CXCL2 levels was

6 already capable of reducing the numbers of monocytes transmigrating through ECs towards

7 SK-OV-3 cells (Fig. 3 h). In summary, the data implicate that the endothelial RBPJ/CXCL2

8 axis contributes to monocyte recruitment into the peritoneum during transcoelomic

9 metastasis.

\section{$11 R$ Rbj in ECs is necessary for tumour-mediated TAM education}

Recruited monocytes differentiate into macrophages which are further educated by

tumour cells to become tumour-promoting macrophages. Macrophage education in EOC is facilitated by hypersensitivity towards IL4, which is induced by tumour cell-mediated cholesterol depletion ${ }^{4}$. We sought to understand whether lack of Rbpj in ECs could influence macrophage phenotypes. To assess this, we isolated newly recruited MN-derived macrophages $\left(\mathrm{CD} 11 \mathrm{~b}^{+} / \mathrm{F} 4 / 80^{+} / \mathrm{CCR} 2^{+}\right)$(Fig. 4a) and obtained their transcriptomic profile by microarray analysis. Gene set enrichment analysis (GSEA) comparing MN-derived macrophages from $R b p j^{i \Delta E C}$ and control tumour-bearing mice revealed that $R b p j$ in ECs is necessary to acquire the typical phenotype of TAM in this model of metastatic EOC (Fig. 4b). As such, tumour cells could not fully educate MN-derived macrophages in mice lacking endothelial Rbpj.

Transcriptomic profiling via Ingenuity Pathway Analysis (IPA) determined that IL4 was the most downregulated signalling pathway in MN-derived macrophages obtained from

$25 R b p j^{i \Delta E C}$ tumour bearing mice (Fig. 4c). IPA and pathway analysis showed that genes 26 important for cholesterol synthesis were downregulated in $\mathrm{MN}$-derived macrophages from $27 R b p j^{i \Delta E C}$ mice (Fig. 4d,e). We then employed a cholesterol homeostasis gene set from TAMs 
1 obtained 21 days after tumour injection ${ }^{4}$, representing EOC-induced cholesterol metabolism

2 in TAMs. GSEA showed that this gene set was significantly enriched in newly recruited

3 macrophages coming from control tumour-bearing mice (Fig. 4f), indicating that Rbpj in ECs

4 is necessary for cholesterol depletion in TAMs.

5 Cholesterol depletion is mediated by tumour cell-secreted high molecular weight

6 hyaluronan (HMW-HA). The interaction with HA receptors, such as CD44, in macrophages

7 leads to cholesterol efflux through $\mathrm{ABC}$ transporters ${ }^{4}$. In order to understand whether Rbpj in

8 ECs could be important for CD44 expression in monocytes, we co-cultured human

9 monocytes with HUVECs lacking RBPJ or with respective controls. Monocytes co-cultured with RBPJ-deficient HUVECs, had less CD44 on their membrane than those incubated with

11 control HUVECs (Fig. 4g). Next, when incubating bone marrow-derived macrophages

12 (BMDMs) with conditioned medium (CM) from immortalized mouse cardiac endothelial cells (MCECs) lacking Rbpj (CRISPR-Cas9 mediated), macrophages expressed less Cd44 (Fig. 4h) and Mmp9 (Fig. 4i), a known CD44 target gene. This indicates that a secreted angiocrine factor regulated by the transcription factor RBPJ in ECs is necessary for the regulation of CD44 in macrophages. To understand whether this would also happen in vivo, $R b p j^{i \Delta E C}$ and control mice were injected with thioglycolate to induce MN-derived macrophage recruitment in the absence of tumour cells. We found that $\mathrm{MN}$-derived macrophages in $R b p j^{i \Delta E C}$ mice expressed significantly less Cd44 than those in control mice (Fig. 4j), confirming that RBPJ in EC is essential for Cd44 regulation in $\mathrm{MN}$-derived macrophages in vivo.

Considering the important role that CXCL2 had in monocyte recruitment in $R b p j^{\mathrm{i} \Delta \mathrm{EC}}$ tumour bearing mice and that higher level of CXCL2 in serum is associated not only with myeloid cell infiltration into the TME, but also with worse prognosis for EOC patients ${ }^{22}$, we decided to analyse its role in regulating CD44 expression. Indeed, when stimulating BMDMs with CXCL2, Cd44 expression was increased (Fig. 4k). Moreover, since CD44 is a

27 membrane-bound receptor, we analysed by immunofluorescence whether also its cellular 28 localization could be altered. We observed that CXCL2 stimulation of BMDMs increased the 
1 presence of CD44 on the plasma membrane, which would consequently increase its

2 accessibility to hyaluronic acid (Fig. 4 I and Suppl. Fig. 4).

4 The TAM gene signature is enriched in human ovarian carcinoma samples with high 5 CXCL2 expression

The data presented so far indicate that the angiocrine factor CXCL2, which is under transcriptional control of Notch/RBPJ signalling, preconditions monocytes to be educated by tumour cells in the TME. In order to evaluate the relevance of this, we analysed CXCL2 and $9 \quad C D 44$ mRNA expression levels in publicly available data sets from the Cancer Genome Atlas 10 (TCGA). Remarkably, the stratification of ovarian cancer patients in $25 \%$ upper (CXCL2 ${ }^{\text {hi }}$ ) 11 and lower $\left(\mathrm{CXCL2}{ }^{\text {low }}\right)$ CXCL2 expression showed that $\mathrm{CXCL2}{ }^{\text {hi }}$ patients displayed significantly more expression of CD44 (Fig. 5a). These data suggest a positive correlation between CXCL2 and CD44 expression in human tumours. Our data indicate that CXCL2mediated CD44 induction reflects the ability of tumour cells to educate TAMs. Therefore, the correlation in expression between CXCL2 and CD44 should in turn have implications on TAM education. Therefore, we performed GSEA comparing CXCL2 ${ }^{\text {hi }}$ and CXCL2 ${ }^{\text {low }}$ patients using the abovementioned TAM signature. We observed that the TAM signature was significantly enriched in CXCL2 ${ }^{\text {hi }}$ patients (Fig. 5b), indicating that the increased CD44 expression in these patients consequently impacts TAM education.

When analysing difference between $\mathrm{CXCL} 2^{\text {hi }}$ and $\mathrm{CXCL} 2^{\text {low }}$ patients, we found an expected upregulation of chemotactic response and myeloid cell recruitment, as shown by gene ontology (GO) term analysis in CXCL2 ${ }^{\text {hi }}$ patients (Suppl. Fig. 5), supporting the role of CXCL2 in controlling the myeloid cell compartment of tumours. Interestingly, we again identified cholesterol metabolism as one of the most downregulated pathways in CXCL2 ${ }^{\text {low }}$

25 patients (Fig. 5c). Instead, CXCL2 ${ }^{\text {hi }}$ patients showed induction of pathways such as lipid and 26 atherosclerosis, thus suggesting opposite profiles (Fig. 5d). Indeed, GSEA showed that the 
same cholesterol metabolism gene set previously employed was significantly enriched in CXCL2 ${ }^{\text {hi }}$ cohort (Fig. 5e), indicating a similar cholesterol depletion as the one occurring in peritoneal macrophages of our mouse model.

\section{TAM education mediated by endothelial Rbpj affects T cell cytotoxicity}

One of the genes downregulated in $\mathrm{MN}$-derived macrophages from $R b p j^{\mathrm{i} \Delta \mathrm{EC}}$ tumourbearing mice is $C d 74$ (Fig. 4b). CXCL2 is a ligand for CXCR2 ${ }^{21}$, and CD44 is part of a receptor complex that contains CD74 and CXCR2 ${ }^{23,24}$. Moreover, CD44 plays an important role in CD74-mediated signal transduction ${ }^{25}$. In silico analysis showed that CD74 interacts closely with CD44 and CXCR2 (Fig. 6a). Besides, CD74 has been reported to be important in TAMs in brain metastasis ${ }^{26}$, and CD74 expression has been associated with worse prognosis in metastatic ovarian cancer $^{27}$. Furthermore, CD74 was associated with an immunosuppressive phenotype in macrophages ${ }^{28}$. We decided to investigate whether the downregulation of CD74 could have a consequence on TAM behaviour. For that, we analysed publicly available data sets where IL4 responses in CD74-deficient macrophages were analysed $^{29}$. GSEA showed that TAM signature was enriched in wild-type macrophages, indicating that CD74 is not only regulated by IL4, but also necessary for TAM education (Fig. 6b). We extracted a signature with the 500 most enriched genes in control compared to CD74-deficient macrophages stimulated with IL4 (CD74-mediated signature), representing a group of genes induced by IL4 through CD74 activation. When comparing newly recruited MN-derived macrophages from our EOC model by GSEA, we found that the CD74-mediated signature was significantly enriched in macrophages isolated from control mice compared to $R b p j^{i \Delta E C}$. This suggests that newly recruited macrophages from $R b p j^{i \Delta E C}$ mice cannot induce their immunosuppressive phenotype due to downregulation of CD74-mediated genes (Fig. 6c). 
To verify the specific effect on the newly recruited macrophages, we repeated the

2 same analysis on resident macrophages (CD11 $\left.\mathrm{b}^{+} / \mathrm{F} 4 / 80^{+} / \mathrm{CCR} 2^{-}\right)$, in which $\mathrm{Cd74}$ is not

3 differentially expressed between $R b p j^{i \Delta \mathrm{EC}}$ tumour-bearing and control mice, and found no

4 enrichment of this gene set in any group, confirming that Rbpj deletion in ECs only affects

5 Cd74 expression in newly recruited MN-derived macrophages (Suppl. Fig. 6). In summary,

6 only macrophages that have crossed the EC barrier as monocytes were affected by the lack

7 of $R b p j$ in the endothelium.

8 It has been previously reported that TME in metastatic ovarian cancer is highly

9 immunosuppressive and infiltration with immune effector cells has little impact in patients'

10 outcome $^{3}$. This immunosuppressive microenvironment has been attributed to TAMs ${ }^{3}$. For

11 this reason, we wanted to test whether impaired TAM education could impact on the

12 phenotype of the TME. We isolated $\mathrm{T}$ cells from tumour-bearing $R b p j^{\mathrm{i \Delta EC}}$ mice and their

13 littermate controls six weeks after intraperitoneal injection of ID8 cells (Fig. 6d). Results

14 demonstrated that $\mathrm{T}$ cells derived from $R b p j^{i \Delta E C}$ mice were more efficient in killing cultured

15 ID8 cells (Fig. 6e). This confirms that impairment in TAM education has a direct impact on T

16 cell cytotoxicity. In addition, the analysis of $\mathrm{T}$ cell populations in peritoneal cavity from

17 tumour-bearing mice revealed that, although the total frequency of $\mathrm{T}$ cells was not changed,

18 the cytotoxic $C D 8^{+} \mathrm{T}$ cell population was significantly increased in $R b p j^{i \Delta \mathrm{EC}}$ mice compared to

19 their littermate controls (Fig. 6f). In summary, our data reveal that Rbpj in ECs is necessary

20 for tumour cell-mediated education of MN-derived macrophages into TAMs (Fig. $\mathbf{6 g}$ ).

\section{Discussion}

Collectively, this study provides evidence about a novel angiocrine axis influencing

24 the tumour immune microenvironment. We show how Notch/RBPJ-mediated transcription in

25 ECs, which is frequently hyperactive in tumours ${ }^{11}$, is required for CXCL2-mediated monocyte 
chemotaxis, induction of CD44 expression on monocytes, and the adoption of a TAM gene signature in metastatic ovarian cancer.

Mice with EC-restricted Rbpj loss had impaired ovarian carcinoma growth in the omentum and peritoneum and lower numbers of $\mathrm{MN}$-derived macrophages in the peritoneal fluid. These macrophages are essential for metastatic ovarian cancer development ${ }^{4,30}$. As such, the endothelium can influence tumour progression and metastasis by altering the immune status of the TME. Specifically, we observed that monocyte recruitment is potentiated, at least in part, through the release of Rbpj-mediated CXCL2 from ECs. Interestingly, higher serum levels of CXCL2 in ovarian cancer patients are associated with myeloid cell infiltration, poor prognosis and chemoresistance ${ }^{31}$. It should be noted that this chemokine has been traditionally associated with the recruitment of neutrophils ${ }^{21}$. However, there is evidence that CXCL2 also plays a role in the regulation of TAMs, especially those derived from monocytes. For instance, the CXCL2 receptor CXCR2 on monocytes and macrophages is important for the education of TAMs in prostate cancer ${ }^{32}$.

CXCR2 blockade has also been shown to re-sensitize ovarian cancer to cisplatin treatment $^{33}$. Here we suggest that the CXCL2/CXCR2 axis might also have a role in the recruitment and education of macrophages in ovarian cancer. By separating newly recruited macrophages from macrophages that have reside in the peritoneal cavity for a longer period, we observed that endothelial RBPJ is necessary for the education into TAMs by tumour cells. Specifically, we report that MN-derived macrophages in contact with ECs lacking RBPJ had a lower expression of the HA receptor CD44. This receptor gets stimulated by HMW-HA produced by tumour cells to induce cholesterol depletion in macrophages, a crucial mechanism by which tumour cells educate $\mathrm{TAMs}^{4}$. Therefore, we propose that through this mechanism ECs can pre-condition MN-derived macrophages and contribute to their immunosuppressive phenotype within the tumour microenvironment. Indeed, the data showed that important genes involved in cholesterol depletion were reduced in $\mathrm{MN}$-derived macrophages from mice lacking RBPJ in their ECs. Consistently, T cells isolated from this 
1 TME were more efficient at killing tumour cells, confirming the reduced immunosuppressive

2 potential of the MN-derived macrophages.

3 In conclusion, we demonstrate that peritoneal ECs are critically involved in the

4 recruitment and education of $\mathrm{MN}$-derived macrophages in ovarian carcinoma.

\section{$6 \quad$ Material and methods}

\section{$7 \quad$ Animal models}

All animal procedures were approved by the local institutional animal care and use committee (RP Karlsruhe, Germany and DKFZ) and performed according to the guidelines

of the local institution and the local government. Female C57BL/6 mice were group-housed under specific pathogen-free barrier conditions.

Administration of tamoxifen diluted in sterile peanut (P2144, Sigma-Aldrich, St. Louis, USA) in 8 to 12-week-old randomized mice was performed by oral gavage once with $100 \mu \mathrm{l}$

14 (1 mg tamoxifen $)^{34}$. Control mice, which did not express Cre ${ }^{\text {ERT2 }}$ were also treated with 15 tamoxifen.

Model of ovarian cancer: Three weeks after gene recombination, $5 \times 10^{6}$ ID8-luciferase

17 (ID8-luc) ovarian cancer cells were administered i.p. in PBS. For peritoneal lavage after sacrificing the mice, $5 \mathrm{ml}$ of ice-cold PBS (Gibco/Thermo Fisher Scientific, NY, USA) was injected i.p., and after a careful massage to mobilize cells, peritoneal fluid was collected. For analysis of ID8-luc tumour growth the cell suspension was centrifuged and supernatant was collected. The cell pellet was suspended in $1 \mathrm{~mL}$ PBS and $100 \mu \mathrm{L}$ were used to determine

22 the luciferase activity. $100 \mu \mathrm{L}$ cell suspension were centrifuged and the cell pellet was suspended in $100 \mu \mathrm{L}$ lysis buffer (Promega) and $20 \mu \mathrm{L}$ of lysed cells were pipetted into white 96-well plate in triplicates. $50 \mu \mathrm{L}$ of LAR substrate (Promega) was added to the lysed cell 
1 BMG Labtech). For analysis of immune cell recruitment into the peritoneal cavity the

2 collected peritoneal cell suspension was centrifuged and red blood cells in the cell pellet

3 were lysed with $1 \mathrm{~mL}$ ACK (Thermo Fisher Scientifics). After washing the cell suspension

4 was counted using Neubauer Counting chamber and $1 \times 10^{6}$ cells were used for flow 5 cytometry staining.

6 Model of peritoneal inflammation: To obtain newly recruited peritoneal macrophages,

$71 \mathrm{~mL}$ thioglycolate (2 mg/mL in $\mathrm{H}_{2} \mathrm{O}$; B2551, Sigma-Aldrich) was injected into the peritoneum

8 three weeks after gene recombination. MN-derived macrophages were isolated 24 hours

9 after thioglycolate injection by their adherence to non-treated plastic petri dishes. Briefly,

10 after incubation of single cells in a petri dish for $30 \mathrm{~min}$ at $37 \mathrm{C}$, non-adherent cells washed 11 away with PBS.

\section{Flow cytometry}

14 For flow cytometry analysis, cells were suspended in $1 \mathrm{~mL}$ PBS with $2 \%$ FCS 15 (Biochrom). Cell suspension was incubated with the different fluorophore-coupled primary 16 antibodies for $20 \mathrm{~min}$ on ice. The following antibodies were used: CD45 (552848), CD11b 17 (552850) and CD4 (560468) and CD8 (557654) from BD Biosciences (Bedford, MA, USA); 18 CD3 (100203), F4/80 (123128), CCR2 (150608), CD44 (mouse and human 103007), CD74 19 (mouse (151005) and human (326811)) from BioLegend (St. Diego, CA, USA), MHCII (4720 5321-80, Life Technologies/Thermo Fisher Scientific, NY, USA) and Tim4 (12-5866-82, Life

21 Technologies/Thermo Fisher Scientific, NY, USA). Concentration of the different antibodies 22 was determined by titration. In the meanwhile, compensation beads (UltraComp eBeads, 23 Thermo Fisher) of the used primary antibodies were prepared. After staining, cells were 24 washed with PBS and stored on ice until acquisition. Acquisition was performed using BD 25 FACSCanto TM II, BD LSR for analysis or Aria for cell sorting (BD Biosciences). 
1 Experiments were analysed using FlowJo Software. Flow cytometer results in percentage

2 were extrapolated to the total number of cells obtained from the cell counting.

3

\section{$4 \quad$ Whole mount staining}

\section{Immunohistochemistry}

The omentum was fixed for 1 hour in 1\% PFA at room temperature and washed with PBS. Tissues were washed three times for $5 \mathrm{~min}$ with permeabilization buffer (PBS, $0.1 \%$ BSA and $0.2 \%$ Triton X-100) and treated with $0.5 \mathrm{~mL}$ blocking buffer (5\% donkey serum diluted PBS-T) for 1 hour at room temperature. Primary antibodies, CD45 (BD 553076) and luciferase (ab185924), were diluted in PBS-T with $2 \%$ donkey serum. Next day, samples were washed three times with PBS- 0.3\% Tween. Afterwards secondary antibodies (1:200) were incubated for 1 hour at room temperature, washed three times with PBS $0.3 \%$ Tween and incubated with DAPI (1:10.000) for 15 min. Next, samples were incubated with clearing solution (FUnGi: $60 \%$ glycerol (vol/vol), $2.5 \mathrm{M}$ fructose, $2.5 \mathrm{M}$ urea, $10 \mathrm{mM}$ Tris Base, $1.0 \mathrm{mM}$ EDTA) at $4{ }^{\circ} \mathrm{C}$ overnight. Coverslips were mounted with FUnGi clearing solution and imaged with a confocal microscope (LSM 710, Carl Zeiss). All images were processed with ZENblue software (Carl Zeiss, Germany). Average mean intensities per image were counted with ImageJ software (NIH, Bethesda, MD, USA).

Paraffin-embedded sections $(3 \mu \mathrm{m})$ were de-paraffinized and re-hydrated in xylene and step-wise reductions in alcohol concentrations. H\&E staining was performed according to standard protocols. Cytokeratin $\mathrm{DAB}$ and $\mathrm{CD} 11 \mathrm{~b}$ : antigen retrieval was performed at $\mathrm{pH} 9$ with citrate buffer. Primary antibodies CD11b (abcam, ab133357, 1:200), and Pancytokeratin (undiluted, ZUC001-125), diluted in blocking solution, were incubated at $4^{\circ} \mathrm{C}$ overnight. After washing, sections were incubated with secondary antibodies coupled with ZytoChem Plus (HRP) Polymer anti-mouse, (ZYTOMED, ZYT-ZUC050-006) and ZytoChem 
1 Plus (AP) Polymer anti-rabbit, (ZYTOMED, ZYT-ZUC031-006) diluted in antibody diluent

2 (Cell Signaling) for one hour at room temperature. Afterwards, slides were treated with DAB

3 Substrat Chromogen, Zytomed, ZYT-DAB057 and AP Red Kit, Zytomed, ZUC001-125.

4 Immunofluorescence staining: primary antibodies: CD31 (Abcam, ab28364, 1:50), VCAM

5 (Vcam, ab134047, 1:200) and aSMA (Sigma-Aldrich, A5228, 1:200) were diluted in blocking

6 solution. Fluorophore-conjugated secondary antibodies (Thermo Fisher Scientific) were

7 diluted in antibody diluent (Cell Signaling) together with isolectin-B4 (Thermo Fisher

8 Scientific, 132450). Images were obtained with slide scanner (Zeiss Axio Sacn.Z1, Carl

9 Zeiss) and a confocal microscope (LSM 710, Carl Zeiss). All images were processed with

10 ZENblue software (Carl Zeiss, Germany). Image quantification were proceeded with ImageJ

11 software (NIH, Bethesda, MD, USA).

\section{Cell culture}

Murine cardiac ECs (MCEC) were purchased from tebu-bio and cultured on gelatincoated surfaces in in DMEM containing $1 \mathrm{~g} / \mathrm{L}$ D-glucose, $5 \%$ FCS, $5 \%$ HEPES, 100 units $/ \mathrm{ml}$ penicillin and $100 \mu \mathrm{g} / \mathrm{ml}$ streptomycin.

HUVEC were grown and maintained until passage 5 in Endopan-3 Growth Medium containing 3\% FCS and supplements (Pan-Biotech). penicillin and $100 \mu \mathrm{g} / \mathrm{ml}$ streptomycin. 
All cell culture experiments were performed in a laminar flow hood and cells cultured

2 at $37^{\circ} \mathrm{C}$ and $95 \%$ relative humidity and $5 \% \mathrm{CO}_{2}$. Cell culture were tested on a regularly basis

3 for mycoplasma contamination periodically and before injecting the tumour cells into the 4 mice.

To study the role of RBPJ in the regulation of Notch target genes, human umbilical

6 vein endothelial cells (HUVECs) were infected with lentivirus constructs for CRISPR/Cas9

7 (Addgene plasmid \#52961, lentiCRISPR v2) induced knock-out for $R b p j^{35}$. After 48 hours,

8 cells were infected with adenovirus (LifeTechnologies; pAD/CMV-V5-DEST) overexpressing

9 GFP as control or N1ICD.

11 Isolation of peripheral blood nuclear cells (PBMC) from buffy coats

Human buffy coats were purchased from blood donation service DRK Mannheim,

13 Germany. Peripheral blood nuclear cells (PBMC) were isolated by gradient centrifugation

14 using Biocoll density solution (L6715; Biochrom). Human buffy coat was diluted 1:1 with PBS

15 and added to the Biocoll density solution. This mixture was centrifuged at $430 \mathrm{~g}$ for 20 min at

16 room temperature. After centrifugation, the white intermediate phase containing leukocytes

17 was collected and washed with PBS. To perform a positive isolation of monocytes, CD14

18 MACS beads were used with the LS column (130-042-402; Milentyi Biotec). The isolation of

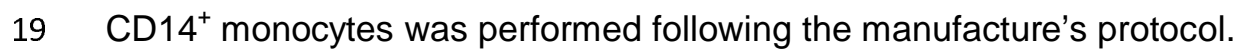

\section{Transwell assay}

Human ovarian cancer cells were seeded at 100,000 cells/ml in $500 \mu \mathrm{l}$ RPMI medium

23 without FCS for 48 hours. For the ECs monolayer, inserts were coated for 2 hours with 2

$24 \mu \mathrm{g} / \mathrm{ml}$ fibronectin (1918-FN-02M; R\&D Systems) in PBS. 50,000 human ECs (RBPJ knock-

25 out or shRNA for CXCL2 (pLKO.1; Sigma Aldrich) and respective controls) were seeded on 
1 top of the insert membrane for 48 hours. To analyse monocyte transmigration, 200,000

$2 \mathrm{CD}_{14}^{+}$cells were stained with carboxyfluorescein succinimidyl ester (CSFE; ThermoFischer)

3 and added onto the endothelial monolayer. The transwell plate was incubated for 2 hours.

4 For the chemotaxis assay with the recombinant proteins, transmigration of 50,000 CD14

5 cells were analysed towards $60 \mathrm{ng} / \mathrm{mL}$ CXCL2 (PeproTech $\mathrm{GmbH}$ ) in RPMI medium without

6 FCS for $30 \mathrm{~min}$. After incubation, the remaining cell suspension in the upper well was

7 aspirated and the transwell was cleaned with a cotton swab. The migrated cells were fixed

8 with $4 \%$ PFA for 20 min at room temperature. Imaging of transwell was performed with Cell

9 Observer (Carl Zeiss). From each transwell five evenly spaced field picture were taken using

$1020 x$ objective and analysis was performed with Image $\mathrm{J}$ software.

\section{Enzyme-linked Immunosorbent Assay (ELISA)}

Protein expression of CXCL2 (MIP-2) was quantified using an enzyme-linked

immunosorbent assay (ELISA; Abcam ab184862). Cell culture supernatant was collected after 24 hours and ELISA was performed following the manufacture's protocol.

\section{Bone marrow-derived macrophages (BMDMs) differentiation}

Mouse macrophages were derived from the bone marrow of wild-type C57BL/6 mice.

Femurs and tibiae were flushed several times with DMEM and collected cells were centrifuged. Bone marrow cells were suspended in media and seeded on $10 \mathrm{~cm}$ petri dishes

21 (Corning). To differentiate these cells into macrophages, $10 \mathrm{ng} / \mathrm{ml} \mathrm{M}$-CSF (PeproTech $22 \mathrm{GmbH}$ ) were added to Dulbecco's modified Eagle's medium (DMEM) (Thermo Fisher) 23 supplemented with $10 \%$ fetal calf serum (FCS) (Biochrom, UK). Differentiation occurred 24 within seven days. Cells were afterwards stimulated with indicated amount of recombinant 25 protein CXCL2 (250-15-20, PeproTech $\mathrm{GmbH})$ in DMEM medium without FCS or conditioned medium of endothelial cells. 


\section{Immunostaining}

BMDMs were cultured in DMEM with 10\% FCS (Biochrom, UK) and 250,000

4 cells/well were seeded into 24-well plates on top of coverslips. BMDMs were treated with 40

$5 \mathrm{ng} / \mathrm{mL}$ CXCL2 (PeproTech $\mathrm{GmbH})$ in DMEM without FCS for 72 hours. Cells were washed

6 with PBS and fixed with 4\% PFA for $10 \mathrm{~min}$. Then, the coverslips were washed three times

7 for 5 minutes with PBS, permeabilized with PBS with $0.1 \%$ Triton X-100 for 10 minutes and

8 blocked for 30 min in blocking buffer (PBS in 5\% FCS with 0.1\% Tween 20 and $100 \mathrm{mM}$

9 glycine) for 1 hour at room temperature. The coverslips were incubated with antibody against

10 CD44 (1:1000) (Abcam, ab124515) and CD45 (1:500) (BD 553076) overnight at $4^{\circ} \mathrm{C}$. The

11 coverslips were rinsed three times with blocking buffer and incubated with a secondary

12 antibody coupled to Alexa Fluor-488 and Alexa Fluor-546 (1:200) for 1 hour. The coverslips

13 were washed and incubated with a DAPI solution before they were washed again.

14 Coverslips were mounted and imaged with a confocal microscope (LSM 710, Carl Zeiss). All

15 images were processed with ZENblack software (Carl Zeiss, Germany).

cDNA synthesis and qPCR

RNA isolation from cell culture was performed using the InnuPrep Mini Kit (Analytik Jena) according manufacture's protocol. RNA isolation form tissue was performed using

20 PicoPure RNA Isolation Kit (Acturus, Life Technology). $1 \mathrm{~mL}$ Trizol was added to the tissue 21 and homogenized for $1 \mathrm{~min}$ and a frequency of $30 / \mathrm{sec}$. After disruption of the tissue, $200 \mu \mathrm{L}$ 22 chloroform were added and mixed by inverting several times followed by a centrifugation 23 step for 15 minutes $12.000 \mathrm{~g}$ at $4^{\circ} \mathrm{C}$. Further RNA isolation steps were performed using the 24 manufacture's protocol. RNA concentration was measured using a Nanodrop 100 (Thermo 25 Fisher Scientific). Reverse transcription of isolated RNA into complementary DNA was 26 performed using High-Capacity cDNA Reverse Transcription Kit (Thermo Fisher Scientific). 
1 Quantitative real-time PCR (qPCR) was performed with SYBR Green PCR mix (Applied

2 Biosystems) on a QuantStudio3 Real-time PCR system (Applied Biosystems). Resulting fold

3 changes were calculated using the $2^{\triangle \triangle C T}$ method and mRNA expression was normalized to

4 the housekeeping gene (Cph for murine and HPRT for human samples).

$5 \quad$ Primers used for mouse genes

\begin{tabular}{|c|c|c|}
\hline Gene Name & Forward Primer & Reverse Primer \\
\hline Cph & ATGGTCAACCCCACCGTG & TTCTTGCTGTCTTTGGAACTTTGTC \\
\hline Ccl1 & CTGCTGCTTGAACACCTTGA & GGTGATTTTGAACCCACGTT \\
\hline Ccl21 & ACCCAAGGCAGTGATGGA & CAGGGTTTGCACATAGCTCA \\
\hline Cxcl2 & CCAACCACCAGGCTACAGG & GCGTCACACTCAAGCTCTG \\
\hline Cxcl12 & GTCCTCTTGCTGTCCAGCTC & TAATTTCGGGTCAATGCACA \\
\hline Cd44 & TCGATTTGAATGTAACCTGCCG & CAGTCCGGGAGATACTGTAGC \\
\hline Mmp9 & CTGGACAGCCAGACACTAAAG & CTCGCGGCAAGTCTTCAGAG \\
\hline
\end{tabular}

$6 \quad$ Primers used for human genes

7

\begin{tabular}{|l|c|c|}
\hline Gene Name & Forward Primer & Reverse Primer \\
\hline $\boldsymbol{H P R T}$ & TGTTGTAGGATATGCCCTTGACT & CTAAGCAGARGGCCACAGAAC \\
\hline $\boldsymbol{C} \boldsymbol{C L 1}$ & CATTTGCGGAGCAAGAGATT & TGCCTCAGCATTTTTCTGTG \\
\hline $\boldsymbol{C} C \boldsymbol{2} \mathbf{2}$ & CAGCCAGATGCAATCAATGCC & TGGAATCCTGAACCCACTTCT \\
\hline $\boldsymbol{C} C L \mathbf{2 1}$ & CCCAGCTATCCTGTTCTTGC & TCAGTCCTCTTGCAGCCTTT \\
\hline $\boldsymbol{C X C L 2}$ & GGCAGAAAGCTTGTCTCAACCC & CTCCTTCAGGAACAGCCACCAA \\
\hline $\boldsymbol{C X C L 5}$ & AGCTGCGTTGCGTTTGTTTAC & TGGCGAACACTTGCAGATTAC \\
\hline $\boldsymbol{C X C L 8}$ & AAGAAACCACCGGAAGGAAC & AAATTTGGGGTGGAAAGGTT \\
\hline $\boldsymbol{C X C L 1 2}$ & ATTCTCAACACTCCAAACTGTGC & ACTTTAGCTTCGGGTCAATGC \\
\hline
\end{tabular}

\section{Cytotoxicity assay}

To analyse the $\mathrm{T}$ cell killing potential by the lactate dehydrogenase (LDH)Cytotoxicity Assay Kit (Ab65393, Abcam), ID8 cells (7,500 ID8 cells in $100 \mu \mathrm{L}$ DMEM medium) were seeded in a 96-well plate one day before $\mathrm{T}$ cell sorting. $\mathrm{T}$ cells were sorted from tumour bearing $R b p j^{i \Delta E C}$ and control mice after six weeks of tumour growth and 10,000 $\mathrm{CD}^{+}$cells were co-cultured with ID8 cells in technical triplicates including untreated control and blank. After overnight incubation, cytotoxicity and killing potential was measured by LDH amount in the cell supernatant using the LDH-Cytotoxicity Assay Kit (Ab65393, Abcam) following the manufacturer's protocol. 
2 In silco analysis of promotor region

3 To determine RBPJ binding $\operatorname{sites}^{36}$ (5'-GTGGGAA-3') in the promotor region of the 4 murine (NM_009140; chr5+:90902580-90903927) and human (NM_002089; chr45 :74100502-74099123) CXCL2-encoding gene, we used ApE plasmid Editor by M. Wayne

6 Davis (https://jorgensen.biology.utah.edu/wayned/ape/).

\section{In silico protein-protein interaction}

We used the Search Tool of Interacting Genes/Proteins database (STRING v11.5) to

10 perform in silico protein-protein interaction analysis ${ }^{37}$. Given CXCR2 protein as input,

11 STRING can search for their neighbour interactors, the proteins that have direct interactions

12 with the inputted proteins; then STRING can generate the PPI network consisting of all these

13 proteins and all the interactions between them. All the interactions between them were

14 derived from high-throughput lab experiments and previous knowledge in curated databases

15 at medium level of confidence (score $\geq 0.40$ ).

\section{Gene set enrichment analysis (GSEA)}

GSEA (Broad Institute) was used to determine if a list of genes (gene signature) was enriched between different groups. A defined list of genes exhibits a statistically significant bias in their distribution (false discovery rate (FDR)) within a ranked gene list using the software GSEA ${ }^{38}$ resulting to an enrichment in one of the compared groups (normalized 
IPA software (Qiagen) was used to identify predicated upstream regulators and

2 differentially regulated pathways in newly recruited macrophages (based on microarray

3 data). For the analysis of data, fold-changes were uploaded to the software. Differentially

4 regulated pathways and upstream regulator analysis was performed from obtained

5 microarray data.

6

7 Pathway analysis

8 Pathway analysis were obtained from public external databases (EnrichR) and 9 analysed as -2log fold changes.

\section{Human ovarian cancer patient RNAseq data analysis}

Human ovarian cancer patient bulk tumour RNA-sequencing data was obtained from

13 the Cancer Genome Atlas (TCGA) database. Stratification in CXCL2 ${ }^{\text {high }}$ and CXCL2 ${ }^{\text {low }}$

14 patients was performed using R Studio software. Patients were assigned to the different

15 groups using CXCL2 expression below the first or higher than the third quartile. Normalised

16 raw counts of CD44 were plotted comparing the two different groups.

\section{Statistical analysis}

Normality was tested when sample size allowed it. Those samples with normal

20 distribution where compared using Students' t-test (with Welch's correction when groups

21 had different sizes). When normality was rejected, Mann-Whitney U-test was used.

22 Comparison analysis was performed with analysis of variance (ANOVA) with Tukey post-test

23 when more than two groups were analysed. Statistical analysis and the generation of the

24 graphs were performed using GraphPad Prism 9 (GraphPad Software, Inc.; La Jolla, CA,

25 USA). 


\section{Schematic Figures}

Schematics were created using BioRender.com.

\section{Acknowledgements}

We thank Ralf Adams (MPI Münster, Germany) for providing Cdh5-CreERT2 mice,

7 we kindly acknowledge Dr. Christiane Opitz for providing the SK-OV-3 cell line and Frances

8 Balkwill (Barts Cancer Institute, London, UK) for providing ID8-luc cells. We thank the Light

9 Microscopy core facility, the Microarray Unit, the Flow Cytometry core facility and animal

10 caretakers of the German Cancer Research Center (DKFZ) for providing excellent services.

11 We would like to thank Damir Krunic (DKFZ, Light Microscopy Core Facility) in particular for

12 his help with FIJI software data analysis.

This work was funded by the Deutsche Forschungsgemeinschaft (DFG) project 394046768 - SFB1366 projects C4, C2 (to A.F.\& A.C.), SPP 1937 (CE 140/2-2 to A.C.),

15 TRR179 (TP07 to A.C.), SFB-TRR156 (B10N to A.C.); the Cooperation Program in Cancer

16 Research of the German Research Cancer Center (DKFZ) and the Israeli Ministry of

17 Science and Technology (MOST) (to A.F.), DFG project 419966437, Deutsche Krebshilfe project 70113888, Ministerio de Ciencia e Innovación (PID2020-115048RB-I00) (to J.R-V.).

19 The Science Ministry of Spain or the Health Ministry (ISCIII) receives support from the EU 20 and its ERDF program. Part of the equipment used in this work has been funded by

21 Generalitat Valenciana and co-financed with ERDF funds (OP ERDF of Comunitat

22 Valenciana 2014-2020). 
Authors declare that they have no competing interests.

\section{References}

101 Ferlay, J. et al. Cancer statistics for the year 2020: An overview. Int J Cancer,

2 Kipps, E., Tan, D. S. \& Kaye, S. B. Meeting the challenge of ascites in ovarian cancer: new avenues for therapy and research. Nat Rev Cancer 13, 273-282, doi:10.1038/nrc3432 (2013).

3 Hensler, M. et al. M2-like macrophages dictate clinically relevant immunosuppression in metastatic ovarian cancer. J Imm unother Cancer 8, doi:10.1136/jitc-2020-000979 (2020).

4 Goossens, P. et al. Membrane Cholesterol Efflux Drives Tumor-Associated Macrophage Reprogramming and Tumor Progression. Cell Metab 29, 1376-1389 e1374, doi:10.1016/j.cmet.2019.02.016 (2019).

5 Noy, R. \& Pollard, J. W. Tumor-associated macrophages: from mechanisms to therapy. Immunity 41, 49-61, doi:10.1016/j.immuni.2014.06.010 (2014).

6 Rafii, S., Butler, J. M. \& Ding, B. S. Angiocrine functions of organ-specific endothelial cells. Nature 529, 316-325, doi:10.1038/nature17040 (2016).

7 Augustin, H. G. \& Koh, G. Y. Organotypic vasculature: From descriptive heterogeneity to functional pathophysiology. Science 357, doi:10.1126/science. aal2379 (2017).

8 Alsina-Sanchis, E., Mulfarth, R. \& Fischer, A. Control of Tumor Progression by Angiocrine Factors. Cancers (Basel) 13, doi:10.3390/cancers13112610 (2021).

9 Zhao, Q. et al. Single-Cell Transcriptome Analyses Reveal Endothelial Cell Heterogeneity in Tumors and Changes following Antiangiogenic Treatment. Cancer Res 78, 2370-2382, doi:10.1158/0008-5472.CAN-17-2728 (2018).

10 Zhao, Q. et al. Heterogeneity and chimerism of endothelial cells revealed by single-cell transcriptome in orthotopic liver tumors. Angiogenesis 23, 581-597, doi:10.1007/s10456020-09727-9 (2020).

11 Wieland, E. et al. Endothelial Notch1 Activity Facilitates Metastasis. Cancer Cell 31, 355-367, doi:10.1016/j.ccell.2017.01.007 (2017).

12 Bray, S. J. Notch signalling in context. Nat Rev Mol Cell Biol 17, 722-735, doi:10.1038/nrm.2016.94 (2016).

13 Limbourg, F. P. et al. Essential role of endothelial Notch1 in angiogenesis. Circulation 111, 1826-1832, doi:10.1161/01.CIR.0000160870.93058.DD (2005).

14 Hasan, S. S. et al. Endothelial Notch signaling controls insulin transport in muscle. EMBO Mol Med 12, e09271, doi:10.15252/emmm.201809271 (2020). 
15 Ramasamy, S. K., Kusumbe, A. P., Wang, L. \& Adams, R. H. Endothelial Notch activity promotes angiogenesis and osteogenesis in bone. Nature 507, 376-380, doi:10.1038/nature13146 (2014).

16 Sorensen, I., Adams, R. H. \& Gossler, A. DLL1-mediated Notch activation regulates endothelial identity in mouse fetal arteries. Blood 113, 5680-5688, doi:10.1182/blood-200808-174508 (2009).

17 Jabs, M. et al. Inhibition of Endothelial Notch Signaling Impairs Fatty Acid Transport and Leads to Metabolic and Vascular Remodeling of the Adult Heart. Circulation 137, 2592-2608, doi:10.1161/CIRCULATIONAHA.117.029733 (2018).

18 Zhu, T. S. et al. Endothelial cells create a stem cell niche in glioblastoma by providing NOTCH ligands that nurture self-renewal of cancer stem-like cells. Cancer Res 71, 6061-6072, doi:10.1158/0008-5472.CAN-10-4269 (2011).

19 Etzerodt, A. et al. Tissue-resident macrophages in omentum promote metastatic spread of ovarian cancer. J Exp Med 217, doi:10.1084/jem.20191869 (2020).

20 Verginelli, F. et al. Activation of an endothelial Notch1-Jagged1 circuit induces VCAM1 expression, an effect amplified by interleukin-1beta. Oncotarget 6, 43216-43229, doi:10.18632/oncotarget.6456 (2015).

21 Jablonska, J., Wu, C. F., Andzinski, L., Leschner, S. \& Weiss, S. CXCR2-mediated tumorassociated neutrophil recruitment is regulated by IFN-beta. Int J Cancer 134, 1346-1358, doi:10.1002/ijc.28551 (2014).

22 Taki, M. et al. Snail promotes ovarian cancer progression by recruiting myeloid-derived suppressor cells via CXCR2 ligand upregulation. Nat Commun 9, 1685, doi:10.1038/s41467018-03966-7 (2018).

23 Gilliver, S. C., Emmerson, E., Bernhagen, J. \& Hardman, M. J. MIF: a key player in cutaneous biology and wound healing. Exp Dermatol 20, 1-6, doi:10.1111/j.1600-0625.2010.01194.x (2011).

24 Alban, T. J. et al. Glioblastoma Myeloid-Derived Suppressor Cell Subsets Express Differential Macrophage Migration Inhibitory Factor Receptor Profiles That Can Be Targeted to Reduce Immune Suppression. Front Immunol 11, 1191, doi:10.3389/fimmu.2020.01191 (2020).

25 Shi, X. et al. CD44 is the signaling component of the macrophage migration inhibitory factorCD74 receptor complex. Immunity 25, 595-606, doi:10.1016/j.immuni.2006.08.020 (2006).

26 Priego, N. et al. STAT3 labels a subpopulation of reactive astrocytes required for brain metastasis. Nat Med 24, 1024-1035, doi:10.1038/s41591-018-0044-4 (2018).

27 Cortes, M. et al. Tumor-associated macrophages (TAMs) depend on ZEB1 for their cancerpromoting roles. $E M B O J$ J6, 3336-3355, doi:10.15252/embj.201797345 (2017).

28 Figueiredo, C. R. et al. Blockade of MIF-CD74 Signalling on Macrophages and Dendritic Cells Restores the Antitumour Immune Response Against Metastatic Melanoma. Front Immunol 9, 1132, doi:10.3389/fimmu.2018.01132 (2018).

29 Przybyl, L. et al. CD74-Downregulation of Placental Macrophage-Trophoblastic Interactions in Preeclampsia. Circ Res 119, 55-68, doi:10.1161/CIRCRESAHA.116.308304 (2016).

30 Moughon, D. L. et al. Macrophage Blockade Using CSF1R Inhibitors Reverses the Vascular Leakage Underlying Malignant Ascites in Late-Stage Epithelial Ovarian Cancer. Cancer Res 75, 4742-4752, doi:10.1158/0008-5472.CAN-14-3373 (2015).

31 Zhang, F., Jiang, J., Xu, B., Xu, Y. \& Wu, C. Over-expression of CXCL2 is associated with poor prognosis in patients with ovarian cancer. Medicine (Baltimore) 100, e24125, doi:10.1097/MD.0000000000024125 (2021).

32 Di Mitri, D. et al. Re-education of Tumor-Associated Macrophages by CXCR2 Blockade Drives Senescence and Tumor Inhibition in Advanced Prostate Cancer. Cell Rep 28, 2156-2168 e2155, doi:10.1016/j.celrep.2019.07.068 (2019). 
133 Henriques, T. B. et al. Inhibition of CXCR2 plays a pivotal role in re-sensitizing ovarian cancer 2 to cisplatin treatment. Aging (Albany NY) 13, 13405-13420, doi:10.18632/aging.203074 (2021).

34 Alsina-Sanchis, E. et al. Intraperitoneal Oil Application Causes Local Inflammation with Depletion of Resident Peritoneal Macrophages. Mol Cancer Res 19, 288-300, doi:10.1158/1541-7786.MCR-20-0650 (2021).

35 Sanjana, N. E., Shalem, O. \& Zhang, F. Improved vectors and genome-wide libraries for CRISPR screening. Nat Methods 11, 783-784, doi:10.1038/nmeth.3047 (2014).

36 Lake, R. J., Tsai, P. F., Choi, I., Won, K. J. \& Fan, H. Y. RBPJ, the major transcriptional effector of Notch signaling, remains associated with chromatin throughout mitosis, suggesting a role in mitotic bookmarking. PLoS Genet 10, e1004204, doi:10.1371/journal.pgen.1004204 (2014).

37 Szklarczyk, D. et al. The STRING database in 2021: customizable protein-protein networks, and functional characterization of user-uploaded gene/measurement sets. Nucleic Acids Res 49, D605-D612, doi:10.1093/nar/gkaa1074 (2021).

38 Subramanian, A. et al. Gene set enrichment analysis: a knowledge-based approach for interpreting genome-wide expression profiles. Proc Natl Acad Sci U S A 102, 15545-15550, doi:10.1073/pnas.0506580102 (2005).

39 de Vos van Steenwijk, P. J. et al. Tumor-infiltrating CD14-positive myeloid cells and CD8positive T-cells prolong survival in patients with cervical carcinoma. Int J Cancer 133, 28842894, doi:10.1002/ijc. 28309 (2013). 


\section{Figure Legends}

2 Figure 1. Reduced tumour burden in omentum and peritoneum of $R b p j^{i \Delta E C}$ mice.

3 a, Model for the spread and proliferation of epithelial ovarian cancer (EOC) cells in the

4 omentum. b, Schematic illustration of oral tamoxifen administration and metastatic EOC

5 protocol. c, Representative pictures of ID8 tumour-bearing mouse omentum and microscopic

6 images of omentum stained with H\&E. Asterisk indicates pancreas. Scale bar, $2 \mathrm{~mm}$. d,

7 Representative images of immunohistochemistry staining for CD31 (white) and DAPI (blue)

8 in omentum of ID8 tumour-bearing $R b p j^{i \mathrm{EC}}$ and control mice. At four weeks after tumour

9 injection. Scale bar, $50 \mu \mathrm{m}$. Quantification of vessel density. Bar graphs show mean $\pm S D$;

10 two-tailed, Welch's corrected t-test. e, Representative images of immunohistochemistry DAB

11 cytokeratin (brown) staining. Scale bar, $20 \mu \mathrm{m}$. Tumour burden quantification of control

$12(\mathrm{n}=12)$ and $R b p j^{i \Delta \mathrm{EC}}$ mice $(\mathrm{n}=9)$. Bar graphs show mean $\pm S D$; two-tailed, Welch's corrected t-

13 test $\mathbf{f}$, Luciferase activity in peritoneal cavity of tumour-bearing $R b p j^{i \Delta E C}$ compared to control

14 mice four weeks after tumour injection. Quantification of luciferase levels in control $(n=12)$

15 and $R_{b p j}{ }^{i \Delta E C}(\mathrm{n}=10)$. Bar graphs show mean $\pm S D$; two-tailed, Welch's corrected t-test.

17 Figure 2: Reduced MN-derived macrophage recruitment in metastatic EOC of $R b p j^{i \Delta E C}$

18 mice.

a, Representative images of whole mount staining of tumour nodules of the omentum for luciferase (red), CD45 (green) and DAPI (blue) four weeks after tumour injection in $R b p j^{i \Delta E C}$ and control mice. Scale bar, $20 \mu \mathrm{m}$. Quantification of infiltrating immune cells $\left(C D 45^{+}\right)$into

22 tumour nodules (luciferase ${ }^{+}$). Bar graphs show mean $\pm S D$; two-tailed, unpaired Mann-

23 Whitney U-test. b, Representative images of tumour infiltrating myeloid cells, CD11 $\mathbf{b}^{+}$(pink)

24 in tumour areas, cytokeratin (brown) from $R b p j^{\mathrm{i} \Delta \mathrm{EC}}$ and control mice four weeks after tumour

25 injection. Scale bar, $50 \mu \mathrm{m}$. Quantification of CD11 $\mathrm{b}^{+}$infiltrating cells area normalized by

26 tumour area. Bar graphs show mean $\pm S D$; two-tailed, Welch's corrected t-test. Analysis of 
1 myeloid cells within the peritoneal cavity after four weeks of tumour growth of $R b p j^{i \mathrm{EC}}$ and

2 control mice by flow cytometry. Percentage of $\mathbf{c}$, myeloid cells (CD45, CD11 $\left.b^{\text {high }}\right)$ and $\mathbf{d}$,

3 macrophages $\left(\mathrm{CD} 45^{+}, \mathrm{CD} 11 \mathrm{~b}^{\text {high }}, \mathrm{F} 4 / 80^{+}\right)$relative to alive cells. Bar graphs show mean $\pm \mathrm{SD}$;

4 two-tailed, unpaired Mann-Whitney U-test. e, Representative flow cytometer plot of

5 monocyte-derived macrophages characterization by F4/80 and MHCII expression into LPM,

6 IntPM and SPM in $R b p j^{\mathrm{i} \Delta \mathrm{EC}}$ mice compared to controls and their quantification. Bar graphs

7 show mean \pm SD; two-tailed, unpaired Mann-Whitney U-test. f, Quantification of newly

8 recruited monocyte-derived macrophages $\left(\mathrm{Tim}^{-} \mathrm{CCR}^{+}\right)$. Bar graphs show mean $\pm \mathrm{SD}$; two-

9 tailed, unpaired Mann-Whitney U-test. g, Scheme of transwell assay with human CD14 ${ }^{+}$

10 monocyte through a monolayer of human umbilical vein endothelial cells (HUVECs) towards

11 human ovarian cancer cells (SK-OV-3). h, Analysis of cell tracer carboxyfluorescein

12 succinimidyl ester (CFSE) stained migrated $\mathrm{CD}_{14}^{+}$monocyte through monolayer of HUVECs

13 with RBPJ KO and their representative images. Bar graphs show mean $\pm S D$; two-tailed, T-

14 student's t-test.

Figure 3. Endothelial Notch-meditated recruitment via CXCL2.

a, Quantification of mRNA expression of CCL1, CCL21, CXCL12, and CXCL2 upon N1ICD overexpression, knock-out of RBPJ or their combination in HUVECs. Bar graphs show n-fold

19 vs GFP transduced as mean $\pm S D$; two-tailed, unpaired Mann-Whitney U-test. b,

20 Quantification of mRNA expression in whole peritoneal fat tissue from $R b p j^{i \Delta E C}$ and control

21 mice. Bar graphs show mean $\pm S D$; two-tailed, unpaired Mann-Whitney U-test. c,

22 Quantification of mRNA expression in whole peritoneal fat tissue from ecN1ICD and control

23 mice. Bar graphs show mean $\pm S D$; two-tailed, unpaired Mann-Whitney U-test. d, Scheme of

24 RBPJ binding in CXCL2 promotor region in murine and human genes. e, Enzyme-linked

25 immunosorbent assay (ELISA) of CXCL2 of cell culture supernatant from HUVECs infected

26 with N1ICD and GFP as control. Bar graphs show mean \pm SD; two-tailed, unpaired Mann-

27 Whitney U-test. f, Analysis of cell tracer carboxyfluorescein succinimidyl ester (CFSE) 
stained migrated CD14 ${ }^{+}$monocyte towards CXCL2 cytokine. Bar graphs show mean \pm SD; two-tailed, unpaired Mann-Whitney U-test. g ELISA of CXCL2 of HUVECs with knock down of CXCL2. Bar graphs show mean $\pm S D$; two-tailed, unpaired Mann-Whitney U-test. $\mathbf{h}$

Scheme of transwell assay with human $\mathrm{CD} 14^{+}$monocyte through a monolayer of human umbilical vein endothelial cells (HUVECs) towards human ovarian cancer cells (SK-OV-3).

Analysis of cell tracer carboxyfluorescein succinimidyl ester (CFSE) stained migrated CD14 ${ }^{+}$ monocyte through monolayer of HUVECs with knock down of CXCL2. Bar graphs show mean $\pm S D$; two-tailed, T-student's t-test.

\section{Figure 4. Essential role of endothelial rbpj for tumour-mediated TAM education.}

a, Schematic illustration of sorting of newly recruited monocyte-derived macrophages for microarray analysis after four weeks of tumour growth in $R b p j^{i \Delta \mathrm{EC}}$ and control mice. $\mathbf{b}$, Gene set enrichment analysis (GSEA) of the tumour associated-macrophages (TAM) signature in recruited macrophages from $R b p j^{i \Delta E C}$ vs. control mice compared with 20 most differentially regulated genes. c, Ingenuity pathways analysis (IPA) for upstream regulator in recruited macrophages from $R b p j^{i \Delta E C}$ and control mice. Analysis of significant differentially regulated pathways by d, ingenuity pathways (IPA) and e, pathway analysis. f, GSEA of cholesterol homeostasis in recruited macrophages from $R b p j^{i \Delta E C}$ vs. control mice with 20 most differentially regulated genes. g, Mean fluorescence intensity (MFI) of CD44 expression of human monocytes co-cultured with human ECs carrying an RBPJ knock-out. Bar graphs show mean $\pm S D$; two-tailed, unpaired Mann-Whitney U-test. Quantification relative to control from Bone marrow-derived macrophages (BMDMs) after stimulation with conditioned medium from immortalized mouse cardiac ECs (MCECs) with Rbpj knock-out and control of h, Cd44 and i, Mmp9 mRNA expression. Bar graphs show mean $\pm S D$; two-tailed, paired students T-test. j, Quantification of mRNA expression of Cd44 from macrophages isolated from peritoneal lavage of $R b p j^{i \Delta E C}$ mice after 24 hours of thioglycollate i.p. injection relative to control. Bar graphs show mean $\pm S D$; two-tailed, unpaired students T-test. k, BMDMs control 
and stimulated with CXCL2 (40ng/ml) for 72 hours. Quantification of mRNA expression of CD44 relative to control. Bar graphs show mean $\pm S D$; two-tailed, paired students T-test. I, Representative images of BMDMs stained with CD44 (white), CD45 (red) and DAPI (blue) of control and stimulated with CXCL2 (40ng/ml) for 72 hours.

Figure 5. CXCL2 expression in ovarian cancer patients correlates with CD44 expression and TAM education.

a, Analysis of $C D 44$ raw counts in $\operatorname{CXCL2}^{\text {hi }}(n=97)$ vs $C X C l 2^{\text {low }}(n=100)$ patient stratification of publicly available bulk tumour of human ovarian cancer from the Cancer Genome Atlas (TCGA) database using R Studio software. Bar graphs show median; two-tailed, unpaired Tstudent's t-test. b, GSEA of the tumour associated-macrophage (TAM) signature in CXCL2 $2^{\text {low }}$ vs. CXCL2 $2^{\text {high }}$ ovarian cancer patients from the TCGA database. Analysis of KEGG pathways of $\mathbf{c}, \mathrm{CXCL} 2^{\text {low }}$ and $\mathbf{d}, \mathrm{CXCL} 2^{\text {high }}$ patients fold changes and shown as Log2-ratio from extracted p-values. e, GSEA of cholesterol homeostasis in CXCL2 ${ }^{\text {low }}$ vs. CXCL2 ${ }^{\text {high }}$ ovarian cancer patients from the TCGA database.

Figure 6. Loss of endothelial Rbpj increases cytotoxic potential and proportion of cytotoxic T cells in peritoneal lavage of tumour-bearing mice.

a, In silico protein-protein interaction analysis shows the association between CXCR2, CD44 and CD74. Results were mapped with CXCR2 as query protein using the STRING database. Protein-protein results are obtained using medium confidence interaction score (0.400). Line thickness of network edges indicates the strength of data support. b, GSEA of publicly available data (E-MTAB-3309) of TAM signature in WT vs. CD74 knock-out (KO) IL-4treated BMDMs with 10 most differentially regulated gene extracted from. c, GSEA of CD74mediated gene signature in recruited macrophages from $R b p j^{i \Delta \mathrm{EC}}$ vs. control mice with 10 most differentially regulated genes. $\mathbf{d}$, Schematic illustration of T cell sorting for cytotoxicity 
assay after six weeks of tumour growth. e, Lactate dehydrogenase (LDH)-cytotoxicity assay

2 of sorted $\mathrm{CD}^{+} \mathrm{T}$ cells and incubation with in vitro cultivated murine ovarian cancer cells

3 (ID8) measured by absorbance at 450nm including blank correction. Bar graphs show

4 mean $\pm S D$; two-tailed, unpaired Mann-Whitney U-test. f, Percentage of CD ${ }^{+}$cells (relative to

$5 \mathrm{CD}^{+} 5^{+}$cells) and their proportion in $\mathrm{CD}^{+}$and $\mathrm{CD}^{+} \mathrm{T}$ cells (relative to $\mathrm{CD}^{+}$cells) in

6 peritoneal lavage of $R b p j^{\mathrm{i} \mathrm{EC}}$ and control mice 4 weeks after tumour injection. Bar graphs

7 show mean $\pm S D$; two-tailed, unpaired Mann-Whitney U-test. g, Model of endothelial Notch1-

8 dependent recruitment and education of MN-derived macrophages (TAM) into the tumour

9 microenvironment. Tumour cells (TC) activate Notch1 on the tumour endothelium. Activation

10 of endothelial Notch signalling leads to a secretion of angiocrine factors, especially CXCL2,

11 which leads to an increased infiltration of monocyte-derived macrophages (by its receptor

12 CXCR2) into the tumour microenvironment. Loss of endothelial Notch signalling inhibits TC-

13 induced education of TAMs by priming of monocytes (MN) leading to a downregulation of

14 hyaluronan receptor, CD44 as well as pro-tumorigenic receptor CD74 on TAMs. Model

15 created with BioRender.com. 

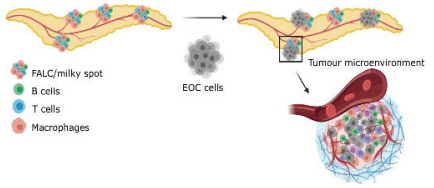

c
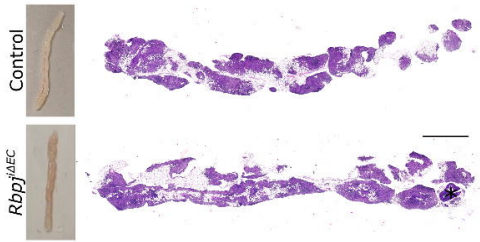

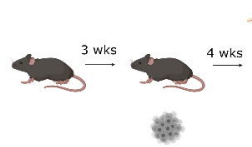

Tamoxifen I.p. injection of

administration murine EOC cells

tumour burden in omentum

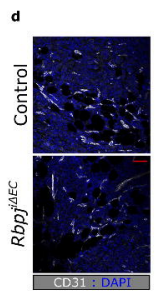

immune cell

e

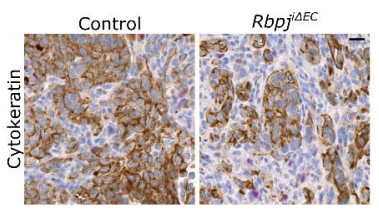

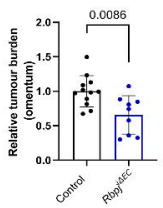

f
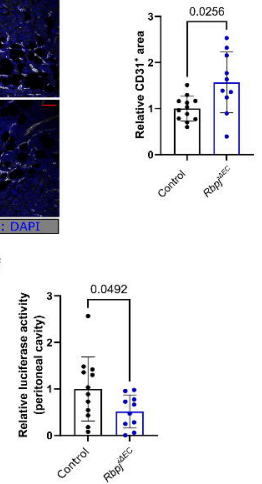

Figure 1. 


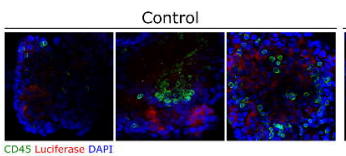

b

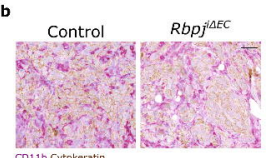

CD11b Cytokeratir

e
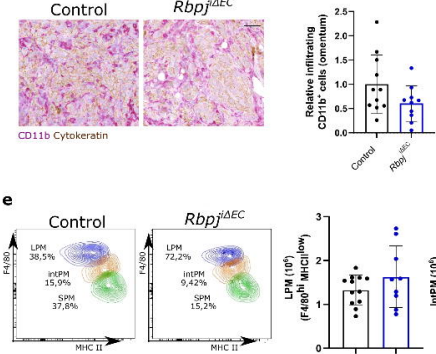

$R b p j^{i \triangle E C}$

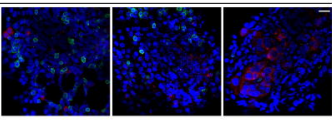

c

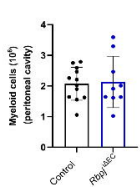

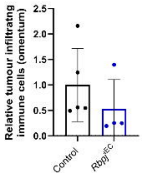

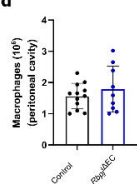

g

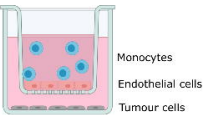

h

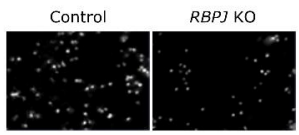

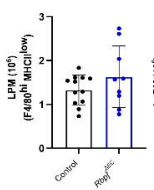
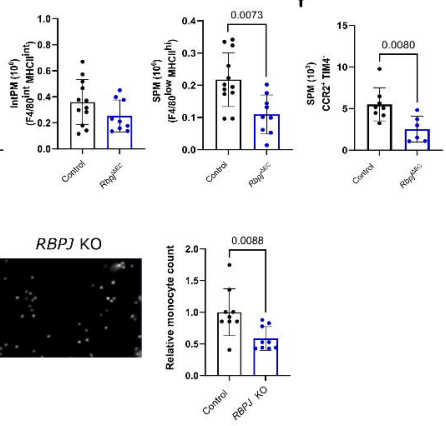

Figure 2. 
a

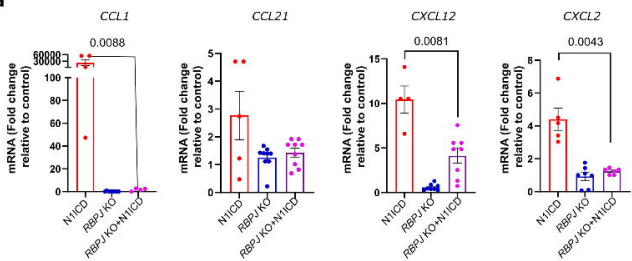

b
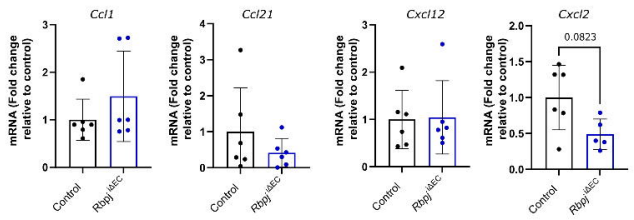

c
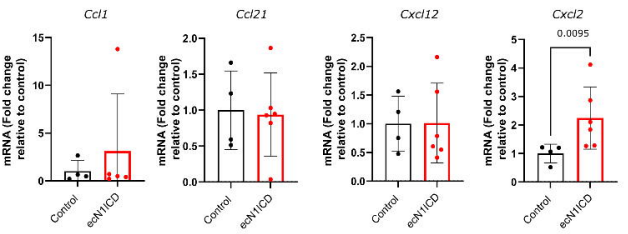

d

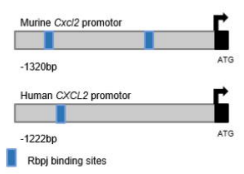

e

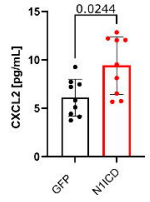

f

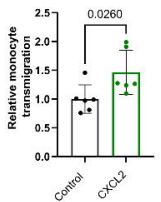

g

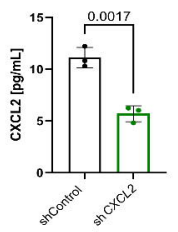

h
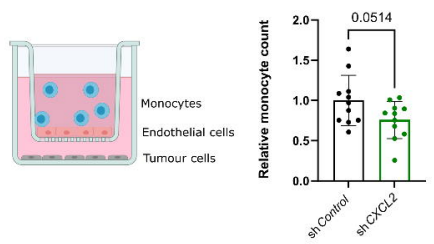

Figure 3 
a

Isolation of newly recruited monocyte-derived macrophages

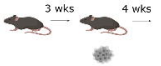

Tamoxifen I.p. injection of Sorting of newly Microarray administration murine EOC oOls

recruited macrophages

d

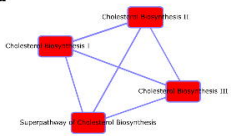

g

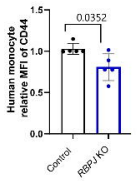

h

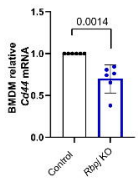

b

acaure Tress

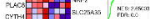

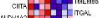

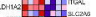

cord
UpstreamRegulator

\begin{tabular}{lll} 
Name & $p$-value & predction \\
\hline IL4 & $4,67^{*} 10^{24}$ & nhibited \\
TGFB1 & $1,15^{*} 10^{-22}$ & inhibited \\
TPS3 & $1,47^{*} 10^{-20}$ & no prediction \\
LPS & $4,89^{*} 10^{20}$ & nhibited \\
IFNG & $2,86^{*} 10^{-19}$ & nhibited
\end{tabular}

IFNG $2,86^{*} 10^{-19}$ inhibited

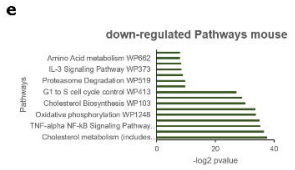

i

j
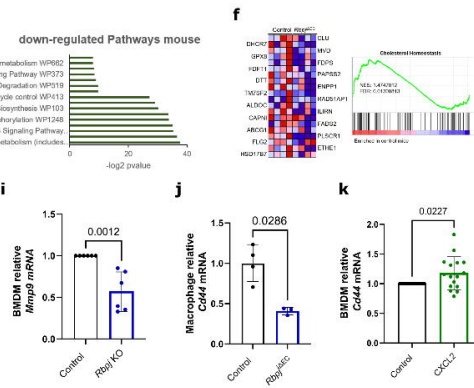

k

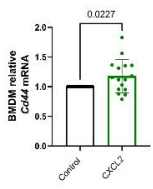

I

Control

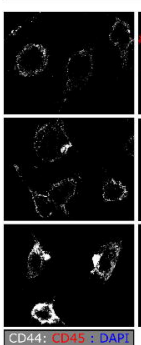

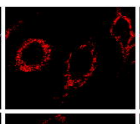
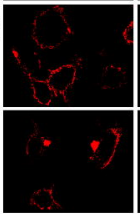

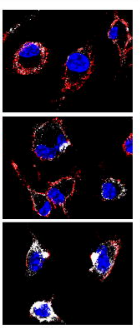

$\mathrm{Cxcl} 2(40 \mathrm{ng} / \mathrm{ml})$

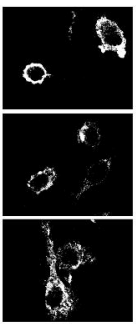

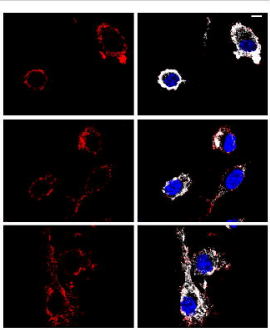

Figure 4. 

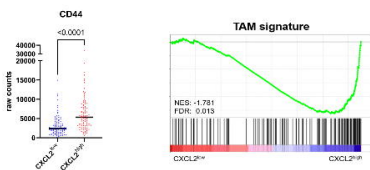

d

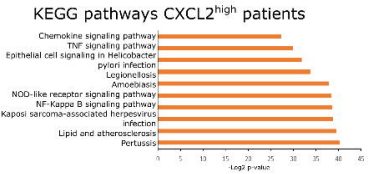

c

\section{KEGG pathways $\mathrm{CXCL} 2^{\text {low }}$ patients}

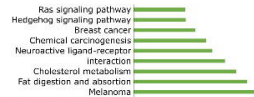

melanoma

Protein digestion and absorptian

e

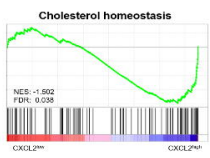

Figure 5. 
a

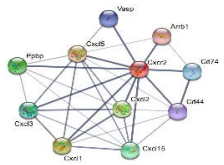

b

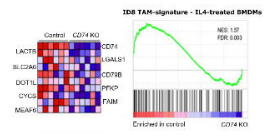

C

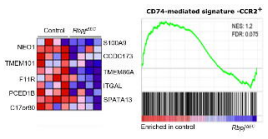

d

Cytotoxicity assay from tumour-bearing mice sorted $T$ cells

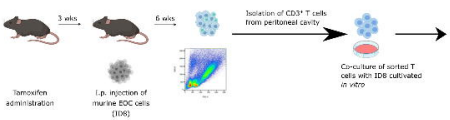

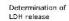

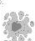

\section{e}

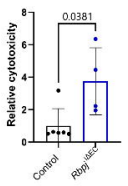

f

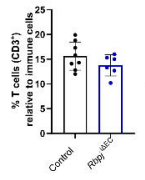

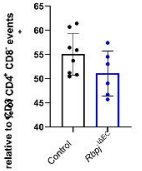

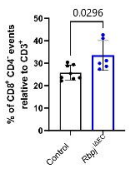

g

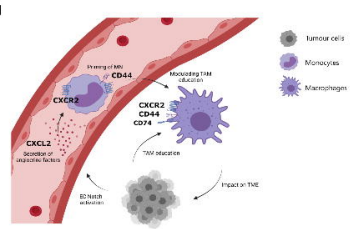

Figure 6 . 